\title{
Surfactant effects in the Landau-Levich problem
}

\author{
By R. KRECHETNIKOV ${ }^{1}$ AND G. M. HOMSY ${ }^{2}$ \\ ${ }^{1}$ California Institute of Technology, Pasadena, CA 91125, USA \\ ${ }^{2}$ University of California, Santa Barbara, CA 93111, USA
}

(Received 6 June 2004 and in revised form 14 December 2005)

In this work we study the classical Landau-Levich problem of dip-coating. While in the clean interface case and in the limit of low capillary numbers it admits an asymptotic solution, its full study has not been conducted. With the help of an efficient numerical algorithm, based on a boundary-integral formulation and the appropriate set of interfacial and inflow boundary conditions, we first study the film thickness behaviour for a clean interface problem. Next, the same algorithm allows us to investigate the response of this system to the presence of soluble surface active matter, which leads to clarification of its role in the flow dynamics. The main conclusion is that pure hydrodynamical modelling of surfactant effects predicts film thinning and therefore is not sufficient to explain the film thickening observed in many experiments.

\section{Introduction}

While many different processes are employed in coating applications, as discussed in the comprehensive book by Kistler \& Schweizer (1997), the simplest - film deposition by withdrawing a substrate from a bath with solution - remains one of the most clear and fundamentally important coating processes. The basic problem is to understand the dependence of the film thickness on the withdrawal speed $U$, the acceleration due to gravity $g$, the size of the domain, and the physical properties of the fluid, i.e. fluid density $\rho$, viscosity $\mu$ and surface tension $\sigma$. This question was first answered by Landau \& Levich (1942) for dip coating from an infinite bath in the lowcapillary-number limit (when surface forces dominate viscous ones). Their analysis, now recognized as a matched asymptotic expansion combined with a lubrication approximation, hinges on the geometrical matching of the constant curvature of the static meniscus to the zero curvature in the thin-film region through a transition region. This yields the classical result,

$$
\bar{h}_{\infty}=0.945 l_{c} C a^{2 / 3}, \quad C a=\frac{\mu U}{\sigma}
$$

where the relevant length scale is the capillary length $l_{c}=\sqrt{\sigma / \rho g}$. The solution (1.1) was successively improved to account for gravity corrections by White \& Tallmadge (1965), but with an incorrect approximation to the normal stress, which was corrected by Spiers, Subbaraman \& Wilkinson (1974). Wilson (1982) put the ad hoc treatments by previous authors on the basis of systematic perturbation theory. For a history of the dip-coating problem, see Tallmadge (1967) for flat substrates, Quéré (1999) on fibre coating, Weinstein \& Ruschak (2004) on general coating flows, and Ruckenstein (2002) for a short summary of ad hoc understanding of coating physics. From the experimental side, the law (1.1) was suggested by Morey (1940) even before the analysis of Landau \& Levich (1942) was published (Of course, the power 2/3 was not known at that time and a general power law was tested using the data). This work, 

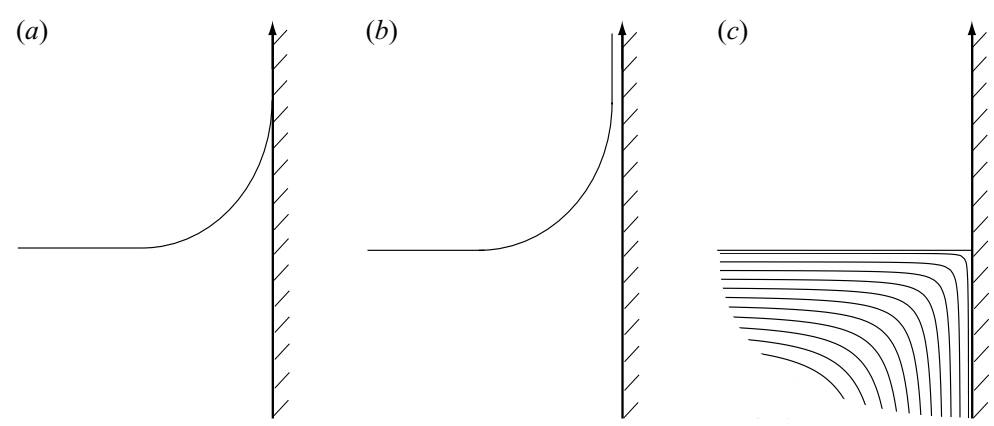

Figure 1. Landau-Levich problem. (a) Static wetting. (b) Dynamic wetting. (c) Far field.

as well as many others (e.g. Deryagin \& Titievskaya 1945) conducted to test and verify the classical law and its generalizations used different variations of gravimetric measurements to determine the film thickness.

Consider a perfectly wetting substrate and an infinite bath of a perfectly wetting liquid as depicted in figure 1(a). This corresponds to what we refer to as the LandauLevich problem, which is an ideal situation, since in reality the bath is always of finite size. When the plate is at rest, the shape of the static meniscus interface is well-known and corresponds to the balance of surface and gravity forces. When the plate starts moving up with speed $U$, the singularity at the contact line is resolved by the pulling up of the interface in this zone by viscous forces and film entrainment (figure $1 b$ ). Assuming that both Reynolds number, based on $l_{c}$ and the speed of withdrawal, and the capillary number are small, the local flow in the meniscus region is creeping and, when viewed in the far field, the flow is as shown in figure 1(c). The meniscus shape stays undistorted (static) except for the small region in which all three forces due to viscosity, surface tension and gravity are in dynamic equilibrium. Therefore, this region is called the dynamic meniscus region. (In this paper we understand the dynamic meniscus to be the region influenced by dynamic effects, such as viscous and Marangoni stresses, i.e. the notion is applicable to the surfactant case as well. For further discussion, see Krechetnikov \& Homsy 2005). The extent of this region (along the plate dimension) $l$ is determined by the balance of viscous and capillary stresses and by matching the curvature in the static and dynamic meniscus regions,

$$
\mu \frac{U}{\bar{h}_{\infty}^{2}} \sim \frac{\sigma / l_{c}}{l}, \quad \frac{\bar{h}_{\infty}}{l^{2}} \sim l_{c}^{-1} .
$$

Therefore, $l \sim\left(\bar{h}_{\infty} l_{c}\right)^{1 / 2}, \bar{h}_{\infty}$ obeys Landau-Levich law (1.1), and the appropriate scalings for the coordinates $\boldsymbol{x}$, velocity field $\boldsymbol{v}$ and pressure $p$, are

$$
\overline{\boldsymbol{x}}=l_{c} \boldsymbol{x}, \overline{\boldsymbol{v}}=U \boldsymbol{v}, \bar{p}=\sqrt{\rho g \sigma} p .
$$

The flow topology in the Landau-Levich problem (figure $2 a$ ), suggests another point of view: the flow in the corner between the moving solid boundary and the free interface as in figure $1(c)$ admits a local self-similar solution (2.7) discussed below, which corresponds to a reversed solution of a flat plate drawn into a viscous fluid found by Moffatt (1964). This self-similarity, of course, breaks down at the corner and is resolved by the above mentioned balance of viscous and capillary forces resulting in film entrainment, which necessitates a sink-type solution superimposed onto Moffatt's solution. 

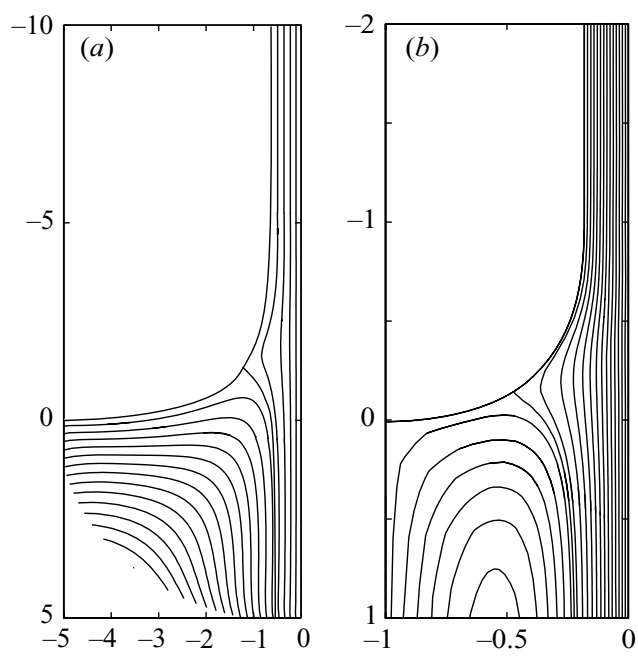

FIGURE 2. Flow field: streamlines patterns at moderate $C a$. (a) Landau-Levich flow.

(b) Bretherton flow.

Therefore, one may consider the Landau-Levich problem as belonging to the class of interfacial singularities that includes Taylor cones, tip-streaming, etc. The physical significance of this $2 / 3$-law is also revealed in a close connection to other problems, such as film deposition in tubes by a penetrating bubble, fibre coating, and a freely suspended film drawn vertically from a reservoir (known as Frankel's law). The first of these problems we refer to as the Bretherton problem, after the asymptotic solution in Bretherton (1961), which is a brother of the Landau-Levich problem, as can be appreciated from figure $2(b)$. However, the fundamental difference in geometry of these two problems (cf. figure 2) - tube versus semi-infinite bath - differentiates them even in the clean interface case. The distinction in geometry leads to a difference in the flow topology - one stagnation point in the Landau-Levich case versus two in the Bretherton problem - which has crucial implications for the surfactant interface case treated here.

The experimental studies of surfactant effects, which are of interest here, are due to Groenveld (1970) and Krechetnikov \& Homsy (2005) for flat substrates; Bretherton (1961) for coating the inner walls of circular tubes; and Carroll \& Lucassen (1973), Ramdane \& Quéré (1997), Quéré \& de Ryck (1998) and Shen et al. (2002) on fibre coating. Historically, in the classical Bretherton problem (cf. Fairbrother \& Stubbs 1935; Taylor 1961), a disagreement was found between the theory by Bretherton (1961) and experiments for $C a<5 \times 10^{-3}$ (which were carried out by Bretherton himself). In this range, the theory underpredicts the measured values of film thickness. This systematic deviation is usually attributed to the presence of surfactants and, as suggested by Schwartz, Princen \& Kiss (1986), is due to accumulation of surfactant in the rear of a finite-length bubble. The common feature of the whole body of experimental observations of the thickening phenomena is the occurrence of very thin films, $\bar{h}_{\infty} \leqslant 20 \mu \mathrm{m}$, for which the deviation is detected.

The more thoroughly studied Bretherton problem reveals some controversy concerning the effects of surfactants. The discrepancy between the theory and experiments by Bretherton (1961) for low capillary numbers was tackled by Ginley \& Radke (1989) for the case of insoluble surfactant, but with constant bulk concentration (which can 
be justified for thick enough films, as will be discussed in $§ 2.3$ ). Their analysis leads to thinning of the film contrary to the known experiments. Alternatively, the discrepancy was ascribed by Ratulowski \& Chang (1990) to the variation in the bulk concentration. This issue was also studied by Wassmuth, Laidlaw \& Coombe (1993) who returned to the assumption of Ginley \& Radke (1989) of constant bulk concentration and constructed a finite-difference solution. The conclusion of their work is that 'strong deviations from the clean-surface... can be either positive or negative depending on the sign of the local surfactant gradient'. Unfortunately, the origin of this variation in sign and connection between the physics and mathematics of interface equations were not uncovered. In the same vein is the work by Ghadiali \& Gaver (2003), who extended the previous numerical analysis to the case of variable bulk concentration using a dual reciprocity boundary-integral method. The extensive numerical data again support the findings of Wassmuth et al. (1993) concerning the influence of surfactants on the film thickness, i.e. either thickening or thinning can occur.

An analogous (even though less rich) body of contradictory literature is observed for the Landau-Levich problem as well. In one of the original works on theoretical explanations of the thickening effect of surface active substances, Groenveld (1970) speculated that the Marangoni effect 'will cause the fluid at the interface in the meniscus region to flow upwards during withdrawal', so that there is no longer a stagnation point at the interface (it is moved to the interior). Later, with no reference to Groenveld (1970), the same simple picture was used by Park (1991) in a lubrication analysis modelling the effect of insoluble surfactant on withdrawal. The key assumption made by Park (1991) is that the dynamic meniscus is restricted to the same scale $l \sim l_{c} C a^{1 / 3}$ as the transition region in the clean interface case, while the rest of the meniscus is both static and uniformly covered by surfactant. The same assumption has been used in other studies of both Landau-Levich and Bretherton problems for trace as well as elevated amounts of surfactant, as done by Stebe \& Barthès-Biesel (1995). As we will show, the meniscus is dynamic in the presence of surfactants on the scale $l \sim l_{c}$ and the stagnation point sits at the interface, thus invalidating all the assumptions made by Groenveld (1970) and Park (1991). The fact that the meniscus is dynamic everywhere, i.e. on the scale $\sim l_{c}$, prevents application of standard perturbation techniques and local analyses, and necessitates a full nonlinear study of an elliptic boundary-value problem.

A closer look at the surfactant dynamics reveals some further difficulties in the attribution of film thickening to Marangoni stresses, at least within the current state of knowledge. The theoretical attempts to justify this effect of thickening through Marangoni stresses in a frame of macroscopic equations has been done for trace amounts of surfactant by Ratulowski \& Chang (1990) for the Bretherton problem with soluble surfactant in the diffusion-limited case and by Park (1991) for the Landau-Levich problem with insoluble surfactant. The common feature of these theories is a lubrication approximation, that is the assumption that the meniscus is dynamic on the same scale as the transition region in the clean interface case, namely $l \sim l_{c} C a^{1 / 3}$. Simple estimates show that this is not always the case. Consider a typical experiment with an aqueous solution of SDS (sodium dodecyl sulfate, the most studied surfactant) at bulk concentrations $C \sim 1 \mathrm{CMC}(8.3 \mathrm{mM})$. When withdrawal speeds are of the order of $1 \mathrm{~cm} \mathrm{~s}^{-1}$, the characteristic time of interface stretching based on the whole meniscus size, $\sim l_{c}$, is $\sim 0.2 \mathrm{~s}$. At the same time, the diffusion length is estimated as a ratio of the interfacial $\Gamma$ and bulk $C$ concentrations, i.e. $\eta_{d} \sim \Gamma / C \sim 1 \mu \mathrm{m}$, and the diffusion time is $t_{\text {diff }} \sim \eta_{d}^{2} / D \sim 10^{-3}$ s versus the adsorption time of $0.2 \mathrm{~s}$ deduced from the kinetic properties of SDS. Since the withdrawal time is of the same order 
as the adsorption time, we can expect that the variation of interfacial concentration in the meniscus is $\delta \Gamma \sim \Gamma$. Therefore, the balance of viscous and Marangoni stresses is not the one assumed by Ratulowski \& Chang (1990), Ramdane \& Quéré (1997), Park (1991) and others, i.e. $\delta \Gamma \sim C a^{2 / 3}$, but rather follows the interfacial sublayer theory of Krechetnikov \& Homsy (2004), which allows significant Marangoni stresses. Therefore, the Marangoni forces in the above parameter regime are significant and not restricted to a short transition region, but rather make the meniscus dynamic on the scale $l \sim l_{c}$. Furthermore, under these conditions the concentration is not dilute, the surfactant transport is sorption-limited, and therefore the picture of Ratulowski \& Chang is not applicable. The analogous lubrication analysis by Ginley \& Radke (1989), which assumes the bulk concentration to be constant and predicts thinning instead of thickening, is subject to the same criticism. These considerations necessitate the full nonlinear analysis of a boundary-value problem.

Thus, for these conditions, which are typical in surfactant coating systems, there is no convincing theory and set of experiments to confirm the viewpoint that the observed thickening is due to Marangoni effects. However, the general belief that this effect is of a purely hydrodynamic nature - the Marangoni stresses pump additional mass flux into the film - is commonly accepted (cf. Quéré 1999). A natural step towards understanding would be to verify by reliable theoretical means that this thickening behaviour is indeed due to Marangoni forces and thus can be accounted for by standard macroscopic equations, which is the primary objective of this paper.

The objectives of our work and the outline of the paper are as follows. In $\S 2$, we formulate the problem mathematically, posing a self-consistent boundaryvalue problem and stressing some subtle mathematical peculiarities with important consequences. In $\S 3$, we provide a self-contained presentation of the method used for the analysis which hinges on the rich experience of other works, but, at the same time, introduces a number of improvements and corrections of the existing literature.

In the application of the method presented in $\S 3$ to the clean interface problem, we study the film thickness functionality for both Landau-Levich and Bretherton problems, and discuss their differences and similarities in $\S 4.1$. Having tested the method with the clean interface problem we introduce surfactant effects in the Landau-Levich problem in $\S 4.2$. In particular, the influence of surfactants on the deposited film thickness and flow topology is investigated. The understanding of the hydrodynamic effects of surface active substances allows us to draw some conclusions towards resolving the long-standing question of the origin of film thickening.

\section{Mathematical formulation}

The geometrical setting for the Landau-Levich problem is sketched in figure 3, which reflects both Cartesian and local systems of coordinates. The use of the scaling (1.2) produces the following evolution equations for the bulk velocity field, $(u, v)=\left(\psi_{y},-\psi_{x}\right)$ :

$$
\begin{aligned}
& \sqrt{L a}\left(u_{t}+u u_{x}+v u_{y}\right)=-p_{x}+1+C a \Delta u, \\
& \sqrt{L a}\left(v_{t}+u v_{x}+v v_{y}\right)=-p_{y}+C a \Delta v,
\end{aligned}
$$

where the two independent parameters are the capillary number and an inertial parameter we refer to as the Landau number,

$$
C a=\frac{\mu U}{\sigma}, \quad L a=\frac{\rho U^{4}}{\sigma g} .
$$




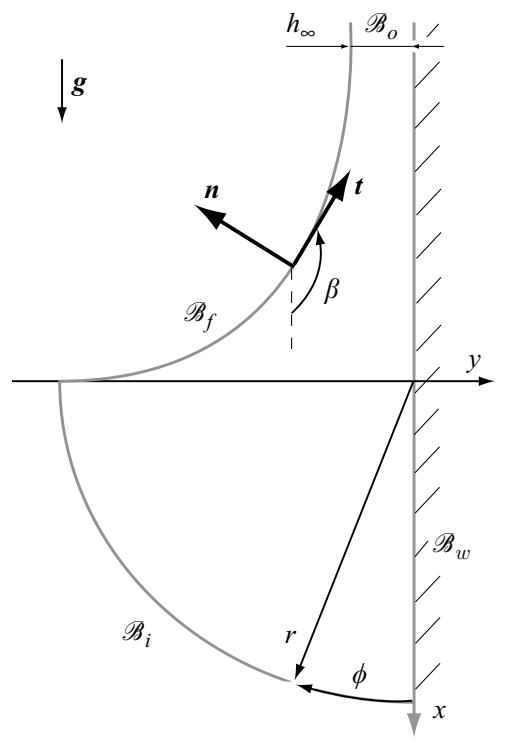

FIGURE 3. Geometrical definition of the problem.

In what follows, the bulk flow will be considered in a Stokes regime, $L a \ll 1$ : this corresponds to a situation when the effective Reynolds number based on the scale of the domain $\mathscr{B}_{i}$ is very small, $R e \ll 1$. Since the appropriate length scale in our case is $l_{c}$, this fact is equivalently expressed in terms of a Bond number, $B o$,

$$
B o \ll C a^{2} L a^{-1} \text {. }
$$

Under these conditions, the solution obeys the biharmonic equation for the streamfunction

$$
\Delta^{2} \psi=0
$$

augmented by an appropriate and consistent set of boundary conditions at each part of the boundary. Once the problem is considered in a steady approximation, without loss of generality we can assign a zero value to the streamfunction at the interface,

$$
\mathscr{B}_{f}: \psi=0 \text {, }
$$

so that on the solid wall we have the regular no-penetration and no-slip conditions

$$
\mathscr{B}_{w}: \psi=\frac{h_{\infty}^{3}}{3 C a}-h_{\infty} \equiv \psi_{w}, \quad \psi_{\boldsymbol{n}}=-1,
$$

and at the outflow portion of the boundary (thin-film region)

$$
\mathscr{B}_{o}: \psi=-\left(h_{\infty}+y\right)+\frac{1}{6 C a}\left(-y^{3}+2 h_{\infty}^{3}-3 h_{\infty} y^{2}\right), \quad \psi_{\boldsymbol{n}}=0 .
$$

The formulation of the remaining boundary conditions at the interface, $\mathscr{B}_{f}$, and those for the inflow region, $\mathscr{B}_{i}$, is more delicate and will be discussed in the following subsections.

\subsection{Inflow boundary conditions}

The inflow boundary condition should provide a realistic velocity profile at $\mathscr{B}_{i}$ and a mass flux exactly equal to the mass flux in the thin film region, $\psi_{w}$. In formulating these conditions we shall use the polar coordinates, $(r, \phi)$, shown in figure 3. 
As mentioned in $\S 1$, the leading-order component of the flow field in this case is a self-similar solution, $\psi=r^{\lambda} f_{\lambda}(\phi)$ with $\lambda=1$, for the corner between a withdrawing solid wall and the free interface, which amounts to the boundary conditions:

$$
\begin{gathered}
\text { solid wall, } \phi=0: f^{\prime}=-1, f=0 ; \\
\text { interface, } \phi=\frac{1}{2} \pi: f^{\prime \prime}=0, f=0 .
\end{gathered}
$$

Their application to the general solution of biharmonic equation, $f=A \cos \phi+$ $B \sin \phi+C \phi \cos \phi+D \phi \sin \phi$, yields

$$
\psi_{s}=r \sin \phi\left(1-\frac{\phi}{\pi / 2}\right),
$$

which indicates that the interfacial speed is $2 / \pi$, and as expected, the flow is not symmetric about $\phi=\pi / 4$. In view of the linearity of the problem this solution can be combined with one responsible for the mass loss due to film deposition in a converging channel. This solution is also of self-similar type, $\psi=r^{\lambda} f_{\lambda}(\phi)$, and satisfies the boundary conditions:

$$
\begin{aligned}
\text { solid wall, } \phi & =0: \psi_{\phi}=0, \quad \psi=\psi_{w} \\
\text { interface, } \phi & =\frac{1}{2} \pi: \psi_{\phi \phi}=0, \quad \psi=0 .
\end{aligned}
$$

Since $\lambda=0$ in this case, the general solution for the biharmonic operator is just $f=A+B \phi+C \phi^{2}+D \phi^{3}$, so that the final solution for this component is

$$
\psi_{c}=\psi_{w}\left\{1-\frac{3}{2}\left(\frac{\phi}{\pi / 2}\right)^{2}+\frac{1}{2}\left(\frac{\phi}{\pi / 2}\right)^{3}\right\} .
$$

The complete condition at $\mathscr{B}_{i}$ is the sum, $\psi=\psi_{s}+\psi_{c}$, i.e. the sum of the Moffatt flow (cf. figure $1 c$ ) and a sink flow.

\subsection{Interface conditions}

We now consider the remaining boundary conditions at the interface, $y=h(\xi)$ with $\xi \equiv x \leqslant 0$ as in figure 3 (and the complete boundary $\bar{\Omega}$ ), which is characterized by the outward normal $\boldsymbol{n}$ and clockwise tangent $\boldsymbol{t}$, defined for the interfacial points $(\xi, h)$ by

$$
\boldsymbol{n}=\frac{\boldsymbol{i} h^{\prime}-\boldsymbol{j}}{\sqrt{1+h^{\prime 2}}}, \quad \boldsymbol{t}=-\frac{\boldsymbol{i}+h^{\prime} \boldsymbol{j}}{\sqrt{1+h^{\prime 2}}},
$$

and parameterized by the arclength, $\mathrm{d} s=-\sqrt{1+h^{\prime 2}} \mathrm{~d} \xi=\mathrm{d} \xi / \cos \beta$, with $\beta$ defined as in figure 3. With these conventions, the normal and tangential stress balances become $\boldsymbol{n} \boldsymbol{T} \boldsymbol{n}=-\sigma \nabla \cdot \boldsymbol{n}$ and $\boldsymbol{t} \boldsymbol{T} \boldsymbol{n}=\boldsymbol{t} \cdot \nabla_{s} \sigma$ respectively, where $\boldsymbol{T}$ is the stress tensor, $\sigma$ is the surface tension and $\nabla_{s}$ is the surface gradient. Written in Cartesian coordinates, these dynamic conditions become

$$
\begin{aligned}
p-\sigma \kappa & =\frac{2 C a}{1+h^{\prime 2}}\left\{h^{\prime}\left(\psi_{x x}-\psi_{y y}\right)-\left(1-h^{\prime 2}\right) \psi_{x y}\right\}, \quad \kappa=\frac{h^{\prime \prime}}{\left(1+h^{\prime 2}\right)^{3 / 2}} \leqslant 0, \\
\frac{\mathrm{d} \sigma}{\mathrm{d} s} & =\frac{C a}{1+h^{\prime 2}}\left\{-4 h^{\prime} \psi_{x y}+\left(1-h^{\prime 2}\right)\left(\psi_{y y}-\psi_{x x}\right)\right\},
\end{aligned}
$$

with an obvious designation for the curvature $\kappa$. Introduction of the local coordinate system $(t, n)$ with origin at some interfacial point $(\xi, h)$ according to $\boldsymbol{r}=\boldsymbol{t} \boldsymbol{t}+n \boldsymbol{n}$ allows 


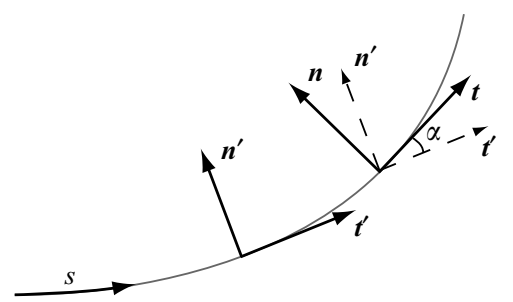

FIGURE 4. On calculating $\psi_{n t t}$.

us to transform the dynamic conditions to the local representation,

$$
\begin{aligned}
p-\sigma \kappa & =-2 \operatorname{Ca}_{n t}, \\
\frac{\mathrm{d} \sigma}{\mathrm{d} s} & =C a\left(\psi_{n n}-\psi_{t t}\right),
\end{aligned}
$$

which in view of the presence of the pressure are still in an inconvenient form for numerical solution by boundary-integral methods. For the purpose of eliminating the pressure, we differentiate the normal component along the tangent vector and use both bulk velocity field equations $(2.1)$ with inertia neglected to eliminate $p_{t}$ to produce

$$
(\sigma \kappa)_{s}=C a\left(\omega_{n}+2 \psi_{n t t}\right)-\frac{1}{\sqrt{1+h^{\prime 2}}},
$$

where $\omega=\Delta \psi$ is a vorticity. Since from a technical standpoint it is much easier to deal with arclength derivatives than with tangential ones, (2.10) must be modified, with particular focus on the term $\psi_{n t t}$. It should be noted that this term was originally treated incorrectly by Kelmanson (1983a): it has nevertheless received wide use in many works, e.g. in Lu \& Chang (1988), Mazouchi \& Homsy (2001) and Goodwin \& Homsy (1991). The mistake was recognized and corrected by Kuiken (1996) (cf. also Mazouchi, Gramlich \& Homsy 2004), but did not receive much appreciation.

This issue of the correct form of the term in question admits an elementary interpretation with the help of figure 4 . Consider two close interfacial points $(\xi, h)$ and $\left(\xi^{\prime}, h^{\prime}\right)$ with the corresponding pairs of unit normal and tangent vectors, $(\boldsymbol{n}, \boldsymbol{t})$ and $\left(\boldsymbol{n}^{\prime}, \boldsymbol{t}^{\prime}\right)$, so that when one moves from $\left(\xi^{\prime}, h^{\prime}\right)$ to $(\xi, h)$ the vectors turn by angle $\alpha$, and are related through

$$
\begin{aligned}
\boldsymbol{n}^{\prime} & =\boldsymbol{n} \cos \alpha+\boldsymbol{t} \sin \alpha, \\
\boldsymbol{t}^{\prime} & =-\boldsymbol{n} \sin \alpha+\boldsymbol{t} \cos \alpha .
\end{aligned}
$$

Therefore, $\psi_{t^{\prime}}=\psi_{t} \cos \alpha-\psi_{n} \sin \alpha$, and the second tangential derivative yields $\psi_{t^{\prime} t^{\prime}}=$ $\cos \alpha\left(\psi_{t t^{\prime}}-\alpha_{t^{\prime}} \psi_{n}\right)+\sin \alpha(\ldots)$, where terms multiplying $\sin \alpha$ are not important since they disappear in the limit $\alpha \rightarrow 0$. Taking into account that $\partial_{t}=\partial_{s}$ and $\partial_{t} \partial_{t^{\prime}}=\partial_{s} \partial_{s^{\prime}}$, the limit $\alpha \rightarrow 0$ furnishes

$$
\psi_{t t}=\psi_{s s}-\alpha_{s} \psi_{n}
$$

Similarly, applying $\partial_{\boldsymbol{t}^{\prime}}^{2}$ to $\psi_{\boldsymbol{n}^{\prime}}=\psi_{\boldsymbol{n}} \cos \alpha+\psi_{\boldsymbol{t}} \sin \alpha$, we obtain

$$
\psi_{n^{\prime} t^{\prime} t^{\prime}}=\cos \alpha\left(-\alpha_{t^{\prime}}^{2} \psi_{n}+\psi_{n t t^{\prime}}+\alpha_{t^{\prime} t^{\prime}} \psi_{t}+2 \alpha_{t^{\prime}} \psi_{t t^{\prime}}\right)+\sin \alpha(\ldots),
$$

which with the use of (2.11) after taking the limit $\alpha \rightarrow 0$, provides the desired expression

$$
\psi_{n t t}=-\alpha_{s}^{2} \psi_{n}+\psi_{n s s}-\alpha_{s} \psi_{n n}+\alpha_{s s} \psi_{s}+2 \alpha_{s} \psi_{s s} .
$$


It is easy to prove that $\alpha_{s}=-\kappa$, so that the normal (2.10) and tangential (2.9b) components of the dynamic interfacial condition with the use of (2.11)-(2.12) become

$$
\begin{aligned}
\sigma \kappa_{s}-\sigma_{s} \kappa & =C a\left\{\omega_{n}+2 \psi_{n s s}-2\left(\kappa \psi_{s}\right)_{s}\right\}+\cos \beta, \\
\sigma_{s} & =C a\left\{\omega-2\left(\psi_{s s}+\kappa \psi_{n}\right)\right\} .
\end{aligned}
$$

While these expressions may appear as obscure, their meaning and sign conventions can be appreciated from two simple problems: surfactant spreading over a flat film and solid-body rotation of an infinite liquid with a bubble around its centre.

The important consequence of $(2.13 b)$ is that the meniscus is always dynamic in the presence of surfactant concentration gradients and this effect becomes more significant with decreasing capillary number. As for the normal stress condition, the presence of the $\omega_{n}$ term explains why one obtains convergence of numerical solutions even with the incorrect version of the term $\psi_{n t t}$ in 2.10: in many physical situations $\omega_{n}$ is the numerically dominant term on the right-hand side of (2.13a) (see figure 7).

\subsection{Surfactant dynamics}

The surface tension in (2.13) is considered as a function of surface excess, $\gamma$, for both soluble and insoluble surfactants. For simplicity, we use the Frumkin equation as a model for material behaviour, which in non-dimensional form is

$$
\sigma=1+\frac{R T \Gamma_{\infty}}{\sigma_{0}} \ln (1-\gamma),
$$

where the surface excess is scaled by the saturation concentration, $\Gamma_{\infty}$, and surface tension by the clean interface value, $\sigma_{0}$. Equation (2.14) exhibits a logarithmic divergence when $\gamma$ approaches saturation, since the work required to compress the surface covered by the finite surfactant molecule cross-section diverges as well. In the case of a soluble surfactant, the sorption kinetics is modelled by a Langmuir isotherm, which at equilibrium, reached far from the moving plate $y \rightarrow-\infty$, in dimensional form is given by

$$
k_{a} C_{0}\left(1-\frac{\Gamma_{0}}{\Gamma_{\infty}}\right)-k_{d} \Gamma_{0}=0,
$$

where $k_{a}$ and $k_{d}$ are adsorption and desorption constants, $\Gamma_{0}$ and $C_{0}$ are interfacial and bulk concentrations at equilibrium. The Frumkin equation in this case becomes the von Szyskowski equation.

The general evolution of surfactant concentration involves both surface convection and diffusion effects. With this convention for scaled variables and with the use of (1.2), the surfactant transport is governed by

$$
\nabla_{s} \cdot\left(\gamma \boldsymbol{v}_{s}\right)+\gamma\left(\nabla_{s} \cdot \boldsymbol{n}\right)\left(\boldsymbol{v}_{s} \cdot \boldsymbol{n}\right)=\frac{1}{P e_{s}} \nabla_{s}^{2} \gamma+j,
$$

where $\boldsymbol{v}_{s}$ is the interfacial velocity vector, $j=S t \kappa_{d}[\zeta(1-\gamma)-\gamma]$ is the sorptive flux, and the non-dimensional parameters are

$$
S t=\frac{k_{a}}{U}, \quad \zeta=\frac{\Gamma_{0} / \Gamma_{\infty}}{1-\Gamma_{0} / \Gamma_{\infty}}, \quad \kappa_{d}=\frac{k_{d}}{k_{a}} l_{c}, \quad P e_{s}=\frac{U l_{c}}{D_{s}} .
$$

The convection terms simplify to

$$
\nabla_{s} \cdot\left(\gamma \boldsymbol{v}_{s}\right)+\gamma\left(\nabla_{s} \cdot \boldsymbol{n}\right)\left(\boldsymbol{v}_{s} \cdot \boldsymbol{n}\right)=\partial_{s}\left(\gamma \psi_{n}\right)-\gamma \kappa \psi_{s} .
$$

Since for steady flow the kinematic condition yields zero velocity normal to interface $\psi_{s}=0$, the contribution to the variation in $\gamma$ due to local changes in interfacial area 
is zero and thus the final form of the surfactant evolution equation is

$$
\frac{\mathrm{d}}{\mathrm{d} s}\left(\gamma \psi_{n}\right)=\frac{1}{P e_{s}} \frac{\mathrm{d}^{2} \gamma}{\mathrm{d} s^{2}}+j,
$$

subject to the equilibrium concentration condition far from the plate and a constant (but unknown) concentration in the thin-film region:

$$
s=0: \quad \gamma=\Gamma_{0} / \Gamma_{\infty}, \quad s=\infty: \quad \gamma_{s}=0 .
$$

The form of the convection term in (2.16) is different from the analogous terms for the bulk evolution equation owing to the lack of a continuity equation for the interfacial velocity. This property has non-trivial consequences for the surfactant distribution, as will be seen in $\S 5$. Since near a stagnation point $s_{0}$, the interfacial velocity is $\psi_{n} \sim\left(s-s_{0}\right)$, the effect of the convective terms is not negligible in the neighbourhood of that point. In the current study, we limit ourselves to the case of film thicknesses for which the diffusion length $\eta_{d} \sim \Gamma_{0} / C_{0}$ defined in $\S 1$ is much smaller than the film thickness, i.e.

$$
\bar{h}_{\infty} \gg \eta_{d},
$$

so that the bulk concentration in the thin-film region is not changed much owing to the adsorption process, and therefore can be assumed to be constant everywhere. This assumption is valid for a wide range of dip-coating conditions including those discussed in $\S 1$. This allows us to disregard the bulk surfactant concentration variations and solve only for the interfacial concentration. As follows from the boundary conditions for $\gamma$, equilibration with the bulk is assumed at both infinite ends of the interface, while in the meniscus region there is dilution of $\gamma$ due to interface stretching competing with sorption kinetics.

\section{Method of solution}

Historically, coating problems have been tackled primarily with the use of finiteelement methods, as in Tanguy, Fortin \& Choplin (1984), who calculated a few solutions for moderate capillary numbers in the case of flat-plate and wire coating, or in Réglat, Labrie \& Tanguy (1993), where a new iterative finite-element methodology is offered. The distinctive feature of the above cited works is the objective of calculating film thickness for a particular physical situation (bath size, fluid properties) without any intention of a more general parametric study. In this context, we should also mention the problem of slot coating treated with the finite-element method by Silliman \& Scriven (1980) and Saito \& Scriven (1981), for example, and the Bretherton problem by Shen \& Udell (1985). The latter problem was also studied by finite differences in the work of Reinelt \& Saffman (1985).

\subsection{Boundary-integral formulation}

The reduction of the problem to the integral formulation (Ladyzhenskaya 1963) offers an alternative approach - the now widespread boundary-integral method (also known as the boundary-element method in the engineering community) - for Stokes flows. As is well known, this method reduces the dimensionality by one, resulting in savings of computational time and storage, and allowing a continuous representation of the solution inside the domain. This method was first successfully applied to flows past a particle of arbitrary shape by Youngren \& Acrivos (1975), extended to free-surface flows in the work of Youngren \& Acrivos (1976), and later used extensively in various drop and bubble problems, including effects of surfactants, as in the work by 
Stone \& Leal (1990). In two dimensions, the advantages of the streamfunction vorticity representation for the biharmonic problem (2.3)

$$
\Delta \psi=\omega, \quad \Delta \omega=0,
$$

and the knowledge of the fundamental solution for Laplace, $\Delta_{p} G_{l}=-\delta(p, q)$, and biharmonic, $\Delta_{p}^{2} G_{b}=-\delta(p, q)$, operators:

$$
G_{l}(p, q)=\frac{1}{2 \pi} \ln \frac{1}{|p-q|}, \quad G_{b}(p, q)=\frac{1}{8 \pi}|p-q|^{2}\left[\ln \frac{1}{|p-q|}+1\right],
$$

respectively, (where $\left.|p-q|=\left[\left(x_{p}-x_{q}\right)^{2}+\left(y_{p}-y_{q}\right)^{2}\right]^{1 / 2}\right)$, were used by Kelmanson $(1983 a, b)$ to extend the boundary-integral method to planar free-surface flows. This generated a pervasive use of the approach in a variety of applications from the already mentioned classical Bretherton problem by Lu \& Chang (1988) to more complicated flows with singular behaviour, such as flows in the vicinity of a dynamic contact line by Goodwin \& Homsy (1991), moving-boundary problems by Mazouchi et al. (2004), Gramlich, Mazouchi \& Homsy (2004), and Stokes flow over topography in Mazouchi \& Homsy (2001).

The continuous and discrete boundary-integral formulations are given in the Appendix. The discretized system (A 5) is the main product of the method and used to solve the biharmonic problem (2.3) along with its boundary conditions. We note that while the equation for interfacial surfactant concentration (2.16) is integrable in quadratures, for numerical efficiency it is solved by finite differences using the Thomas algorithm; in view of the hyperbolic nature of the convective terms in (2.16) they are discretized using upwind differencing to avoid non-physical oscillations.

\subsection{Iteration scheme}

Since in this work we are interested in capillary numbers below unity, $C a<1$, the appropriate iterating procedure is based on the normal stress condition. This fact was established in the work of Silliman \& Scriven (1980), who also found that when the capillary number rises above unity the appropriate iteration equation is the kinematic one. In our case there are two explicit iterative approaches to construct the solution. Before describing each one in detail, we outline the basic relations important for reconstructing the interface. In view of the steep profile of $h(\xi)$ for both the flat bath surface and the thin-film regions, the best parameterization is based on the arclength $s$. It is easy to establish that interfacial slope $\beta$ defined in figure 3 is related to curvature via

$$
\frac{\mathrm{d} \beta}{\mathrm{d} s}=-\kappa,
$$

with boundary conditions $s=0: \beta=\pi / 2$ and $s=\infty: \beta=\pi$, so that $\beta / 2 \pi$ is a rotation index for the portion of the curve between $s=0$ and $s$. The knowledge of $\beta(s)$ allows us to reconstruct the interfacial coordinates,

$$
\frac{\mathrm{d}}{\mathrm{d} s}(\xi, h)=(\cos \beta, \sin \beta),
$$

with obvious boundary conditions at both ends, $s=0: \xi=0, h=-B o$ and $s=\infty: \xi=-\infty, h=-h_{\infty}$. In all iterative methods we first assign some initial guess for the curvature at each node $\kappa^{0}(s)$; then with the help of (3.2)-(3.3) we reconstruct the interfacial coordinates for element ends and nodes; then the solution of the boundaryvalue problem (without the normal stress condition) yields the flow field; this is used 
in obtaining a new surfactant distribution, $\gamma^{j}(s)$, from (2.16) and in deducing a new curvature, $\kappa^{j}(s)$, from one of the iteration schemes described below.

(i) Picard iterations. This approach (however, not in the arclength formulation) was used in the analogous Bretherton problem by Lu \& Chang (1988). Dividing (2.13a) by $\sigma^{2}(s)$ and integrating with respect to $s$ from the origin to an arbitrary point at the interface we obtain a Picard equation, which for the $j$ th iteration assumes the form

$$
\frac{\kappa^{j}(s)}{\sigma(s)}=\left[\frac{\kappa(0)}{\sigma(0)}+\int_{0}^{s} F\left(\widehat{s}, \kappa^{j-1}\right) \mathrm{d} \widehat{s}\right], F\left(s, \kappa^{j-1}\right)=C a\left\{\omega_{n}+2 \psi_{n s s}\right\}+\cos \beta,
$$

where $F\left(s, \kappa^{j-1}\right)$ indicates a functional dependence on the curvature from the previous, $j-1$, iteration. Equation (3.4) is first integrated up to $s=\infty$ to find $\kappa(0)$ from the condition $\kappa(\infty)=0$. Ideally, for $B o=\infty$ there should be $\kappa(0)=0$, which corresponds to the flat bath interface. The shortcoming of this approach comes from working with the curvature, which might be matched to zero in the thin-film region and correspond to the flat interface, but does not guarantee that the interface is parallel to the wall.

As simulations demonstrated, this approach is extremely sensitive to the initial guess and for $B o>1$ obtaining convergence becomes hopeless. This stimulated us to use a different approach, which fixes not only the curvature value at $s=\infty$, but also imposes the restriction $h_{s}=0$, which presumably should limit the possibilities for trajectories and stabilize iterations.

(ii) Converting the normal stress condition to the second-order ODE. The basic idea of this approach is to introduce more control to the slopes of the interface at both ends, $s=0$ and $s=\infty$. Since the curvature can also be expressed in terms of arclength derivatives of $h(s), \kappa=h_{s s}\left(1-h_{s}^{2}\right)^{-1 / 2}$, the normal stress condition can be reformulated as a two-point second-order boundary-value problem for $H \equiv h_{s}$,

$$
H_{s s}+\left(\frac{H H_{s}}{1-H^{2}}-\frac{\sigma_{s}}{\sigma}\right) H_{s}+\frac{1-H^{2}}{\sigma}=t\left(1-H^{2}\right)^{1 / 2} \frac{C a\left(\omega_{n}+2 \psi_{n s s}\right)}{\sigma},
$$

with the two boundary conditions, $s=0: H=1$ and $s=\infty: H=0$. While the second 'convective' term on the left-hand side of (3.5) might suggest upwind differencing, the fact that the flow field is incompressible suggests a numerical approach appropriate for elliptic equations, i.e. central differencing. Indeed, our experience shows that central differencing does not lead to oscillations. The use of (3.5) instead of (3.4) leads to a more robust numerical algorithm, which works for $B o>1$ as well, and to more accurate resolution of the thin film as compared to the work of Lu \& Chang (1988), whose results for the Bretherton problem involve 30-40\% error.

In both models, equations (3.4)-(3.5) are solved via relaxation

$$
\kappa^{j-1}+r_{\kappa}\left(\kappa^{j}-\kappa^{j-1}\right) \rightarrow \kappa^{j}, \quad \gamma^{j-1}+r_{\gamma}\left(\gamma^{j}-\gamma^{j-1}\right) \rightarrow \gamma^{j},
$$

which is used to adjust the rates of change of curvature and surfactant concentration to that of the flow field. Convergence is usually reached for relaxation parameters $r_{\kappa} \leqslant 10^{-3}$ and $r_{\gamma} \leqslant 10^{-2}$. Instead of using local convergence criteria based on $h_{\infty}$ as in Lu \& Chang (1988), we exploited a global criterion based on the convergence of the curvature at all interfacial nodes, $\sum_{i=1}^{N}\left(\kappa_{i}^{j}-\kappa_{i}^{j-1}\right) / \sum_{i=1}^{N} \kappa_{i}^{j-1} \leqslant 10^{-4}$, which is a stronger criterion, but required a greater number of iterations (but usually less than $\left.10^{3}\right)$. The number of boundary elements was determined from the convergence of the film thickness $h_{\infty}$ with 5\% tolerance, which is usually reached with 150-200 boundary elements. The use of a non-uniform grid with elements concentrated in the dynamic meniscus and thin-film regions does not yield any benefits in terms of convergence 


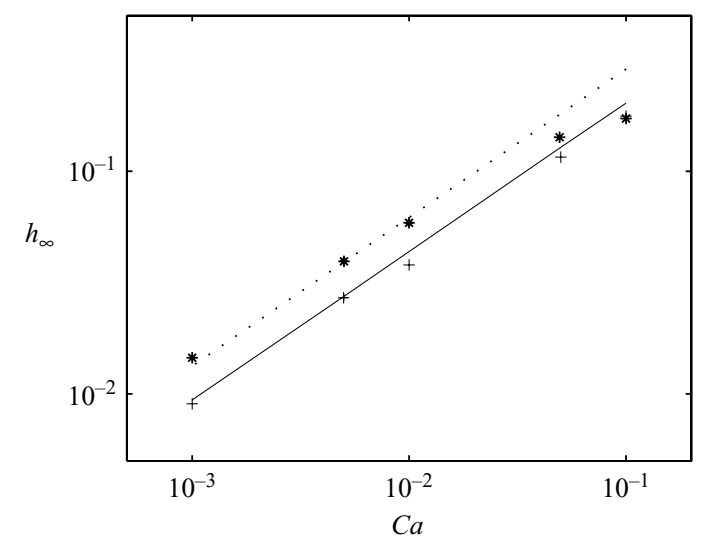

FIGURE 5. Film thickness for the Landau-Levich (solid curve, + ) and Bretherton problems (dashed curve, *).

rate and number of elements. Lastly, the intrinsic limitation of the boundary-integral method to very thin-films should be mentioned - cancellation errors across the thickness imposes a lower limit on the capillary number. The analogous issue is well known in thin-shell problems (cf. Becker 1992).

\section{Results}

\subsection{Clean interface case}

In order to appreciate some of the more intricate physical aspects of the clean LandauLevich problem, we continue the parallel with the Bretherton problem initiated in $\S 1$. In order to compare these two problems in the clearer form, we compare their theoretical single-term asymptotic limits,

$$
h_{\infty}^{B}=1.337 C a^{2 / 3}, \quad h_{\infty}^{L}=0.945 C a^{2 / 3},
$$

with the calculated solutions for $10^{-3}<C a \leqslant 10^{-1}$ in figure 5 .

As seen, appreciable deviation from the theory is observed only when $C a \simeq 10^{-1}$, that is when the asymptotic theory usually fails. Our results for the Bretherton problem are much closer to the asymptotic prediction than those of $\mathrm{Lu} \&$ Chang (1988). At the same time, our calculations for the Landau-Levich problem agree well with the asymptotic solution by Wilson (1982), whose second dominant term, $0.10685 \mathrm{Ca}$, is negative and thus predicts a negative departure from the classical Landau-Levich solution at finite $C a$.

The natural question, "why is the film for the Bretherton problem thicker than that for the Landau-Levich problem at a given $C a$ number?', can be answered by comparing the pressure distribution for these two problems on the same scales of arclength, which is conveniently done by considering the Landau-Levich problem with $B o=1$. In figures 6 and 7, origin of arclength coordinate, $s=0$, corresponds to the left-most interfacial position. Figure 6 , obtained by integrating

$$
\frac{\mathrm{d} p}{\mathrm{~d} s}=C a \omega_{n}+\cos \beta,
$$

indicates that the pressure gradient, directly responsible for the film thickness in the Bretherton problem via $p_{x}=-3\left(1-h_{\infty}\right)$, is larger than that in the Landau-Levich problem. The last term in (4.2) comes from the presence of gravity which is absent in the Bretherton problem. In the ideal Landau-Levich problem, this term makes the 


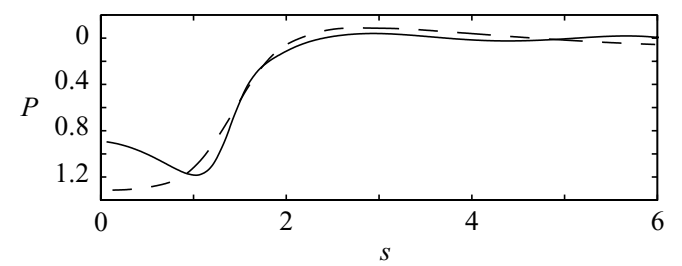

Figure 6. Pressure distribution along the interface for the Landau-Levich (solid curve) and Bretherton (dashed curve) problems. $s$ is an arclength coordinate.

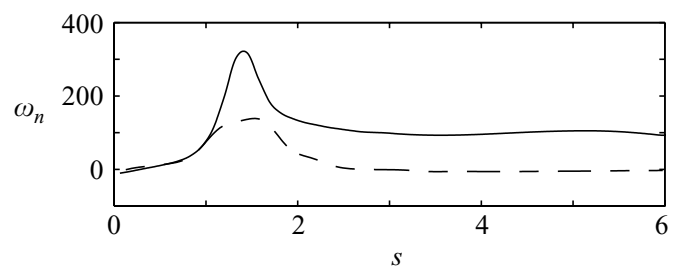

FiguRE 7. Distribution of $\omega_{n}$ along the interface for the Landau-Levich (solid curve) and Bretherton problems (dashed curve). $s$ is an arclength coordinate.

solid line in figure 6 tend to its zero asymptotic value not only in a thin film, but also in the flat bath region.

The distribution of $\omega_{n}$ shown in figure 7 is not only closely connected to the pressure gradient, as seen from (4.2), but is also the leading-order term in the normal stress condition $(2.13 a$ ), for both the Landau-Levich and Bretherton problems. As can be seen, it rises from zero to very large values, which are due to the presence of a stagnation point at the interface in the vicinity of the solid wall. This large magnitude of $\omega_{n}$ explains why Kelmanson's incorrect version of the normal stress condition gives results close to that from the correct version for both the Bretherton and Landau-Levich problems, as it is numerically dominant over other terms in $(2.13 a)$.

\subsection{Surfactant interface case}

As mentioned in $\S 1$, the substantial effect of a surface active substance on the deposited film thickness is usually attributed to the tangential forces due to concentration gradients. To validate this picture, Groenveld (1970) speculated on the absence of the stagnation point at the interface, while Park (1991) invoked the assumptions of a static meniscus and uniform concentration distribution. As the tangential stress condition $(2.13 b)$ indicates, the meniscus is not static in the presence of surfactant. Moreover, our numerical solutions for insoluble surfactant (not shown) confirmed the intuitive understanding that an insoluble surfactant is simply convected away from the solid wall. This is easy to appreciate from the flow topology in figure 2(a), which has a single stagnant point. Thus an insoluble surfactant introduces no influence on the film entrainment dynamics.

As a result, here we study the effect of surfactant on the Landau-Levich problem in the soluble case. To make understanding of the surfactant effect more general, previous workers (cf. Quéré 1999; Shen et al. 2002) developed a measure of the effect of surfactants. Since in many experiments it was found that film thickness increases, a 'thickening factor' was defined as the ratio of the resulting experimental film thickness to a (hypothetical) theoretical one for uniformly distributed surfactant. This has the advantage of separating the hypothesized Marangoni influence from a simple lowering of the surface tension. Given the concentration $\Gamma_{0}$ and capillary 
(a)

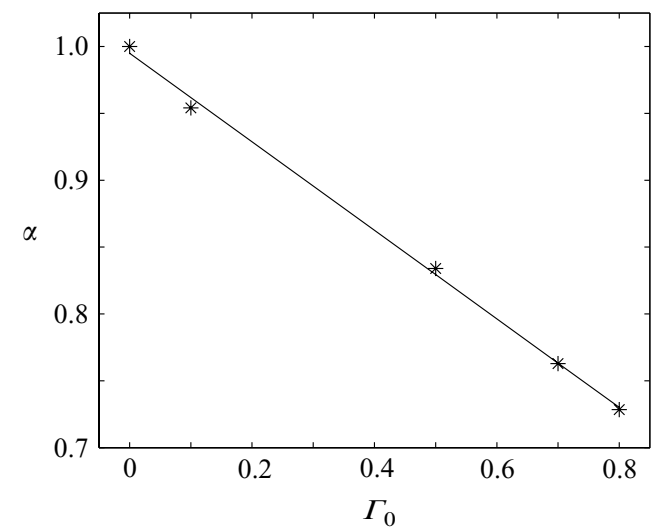

(b)

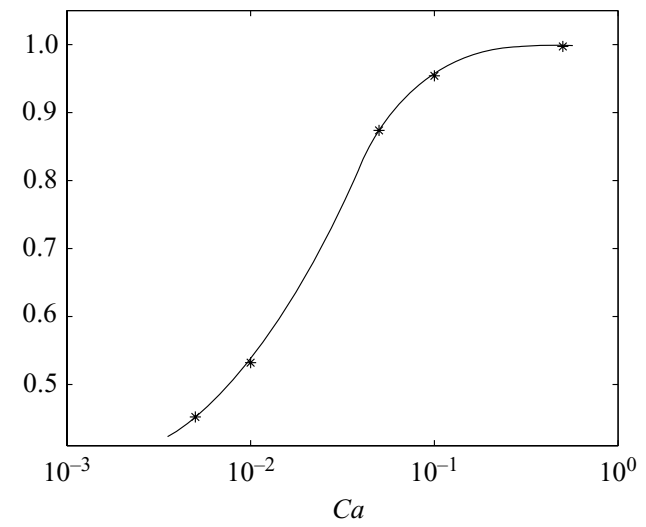

FIgURE 8. Thinning factor. (a) Dependence on $\Gamma_{0}$ for $C a=10^{-1}$. (b) Dependence on $C a$ for $\Gamma_{0}=0.1$.

number, this factor is determined by

$$
\alpha=\bar{h}_{\infty} / \bar{h}_{\infty}^{\text {theory }}, \quad \bar{h}_{\infty}^{\text {theory }}=0.945 \sqrt{\frac{\sigma\left(\Gamma_{0}\right)}{\rho g}}\left(\frac{\mu U}{\sigma\left(\Gamma_{0}\right)}\right)^{2 / 3},
$$

that is $\bar{h}_{\infty}^{\text {theory }}$ contains the effect of lowering the mean surface tension which affects both the capillary length and capillary number. It should be mentioned that from an experimental standpoint this is a virtual variable, obtainable only by theoretical means. In our theoretical analysis, however, we can compute the film thickness corresponding to a uniform distribution of surfactant and, since we are working with non-dimensional variables scaled by the same capillary length for $\Gamma_{0}=0,(4.3)$ becomes

$$
\alpha=h_{\infty} / h_{\infty}^{\text {theory }}, \quad h_{\infty}^{\text {theory }}=h_{\infty}^{\text {theory }}\left(\Gamma_{0}=\text { const }\right) .
$$

It is notable that the film thickness dependence on surface tension for fibre coating and for plate withdrawal is a different power, $2 / 3$ and $1 / 6$, respectively, since in the former the length scale is a fibre radius, while in the latter it is the capillary length. This has some effect on the factor $\alpha$.

Experiments by Ramdane \& Quéré (1997) and Shen et al. (2002) for coating on wires have shown that $\alpha$ is nearly independent of $C a$. This is remarkable, but difficult to rationalize with a general picture of the effect of Marangoni stresses. Analyses by Ratulowski \& Chang (1990) and Park (1991), in spite of their shortcomings, predict that $\alpha=\alpha(\mathrm{Ca})$. Perhaps more significantly, the wider body of experimental results surveyed by Quéré \& de Ryck (1998) also suggest that $\alpha$ is not generally independent of $\mathrm{Ca}$.

With the above definition of the thickening factor $\alpha$ and with the use of the Frumkin equation (2.14) we determined the functionalities, $\alpha\left(\Gamma_{0}\right)$ in figure 8(a) and $\alpha(C a)$ in figure $8(b)$ for fixed capillary number and $\Gamma_{0}$, respectively. Calculations have been performed for a reference set of parameters, $S t=0.5, P e_{s}=10.0, R T \Gamma_{\infty} / \sigma_{0}=0.1$, and $\kappa_{d}=1.0$. Contrary to the experimentally observed thickening, Marangoni effects lead to thinning and, therefore, we refer to $\alpha$ as a 'thinning factor' when $\alpha<1$. Figure $8(b)$ shows that the predicted thinning factor is not independent of $\mathrm{Ca}$. This is easy to understand, since for small speeds of withdrawal (low capillary number) the adsorption compensates the lowering of the surface excess owing to interface 


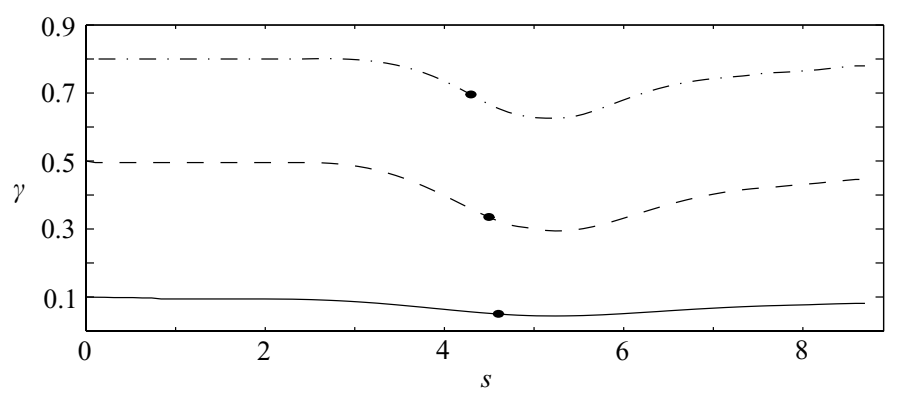

FIGURE 9. Distribution of surfactant along the interface for three different bulk concentrations and $C a=0.1$. Filled circles designate the location of the stagnation point at the interface.

stretching and thus no significant Marangoni stresses can be expected. Thus, we could not expect the same factor $\alpha$ for low and high speeds. However, our measurements of film thickness on a planar substrate (Krechetnikov \& Homsy 2005) also demonstrate no significant variation of $\alpha$ with the speed, thus posing further questions as to the origins of the film thickening.

In order to understand the physical mechanisms leading to thinning, we examine the distribution of surfactant along the interface in figure 9 for different bulk concentrations. In both the thin-film and flat bath interface regions, we observe the expected equilibration with the bulk concentration $C_{0}$, so that $\gamma \rightarrow \Gamma_{0} / \Gamma_{\infty}$. In the meniscus region, there is generation of a new interfacial area and thus interface stretching which leads to dilution of the interfacial surfactant concentration competing with sorption kinetics - therefore we observe a lower $\gamma$ in this region. One would probably expect that since fluid particles stay an infinitely long time in the neighbourhood of a stagnation point, the surface excess should approach its equilibrium value $\Gamma_{0} / \Gamma_{\infty}$. It turns out that it is not true, as follows from figure 9 - the fundamental difference in interfacial concentration convection terms discussed in $\$ 2.3$ from those for bulk concentration leads to this specific behaviour. The net result is the existence of two Marangoni flows - one from the thin film towards the meniscus and the other from the bath towards the plate. The first obviously leads to thinning, while the second seems to support the point of view of Groenveld (1970) that the Marangoni flow contributes the flux to the film. However, the presence of a stagnation point, which persists in this range of parameters, prevents this Marangoni flux from overcoming the thinning Marangoni flow from the film region, the latter apparently being stronger since the stagnation point moves away from the wall as $\Gamma_{0}$ grows. As follows from figure 9, the variation of $\gamma$ grows with an increase of $\Gamma_{0}$ and therefore the Marangoni thinning effect grows as well (cf. figure $8 a$ ).

\section{Summary and conclusions}

In this work we revisited the classical problem of dip-coating first solved asymptotically in the low-capillary-number and clean interface case by Landau \& Levich (1942). With the objective of studying the effect of surfactants on the LandauLevich problem, we revisited the overall mathematical formulation and the numerical algorithm due to Kelmanson (1983a) and Lu \& Chang (1988). This has led to a number of improvements and clarifications: among them the general set of interfacial dynamic conditions in local coordinates, inflow boundary conditions, and a more stable iteration procedure. The method was tested on the clean interface case for both Landau-Levich and Bretherton problems. 
Our results are given for 'incompressible' bulk concentration dynamics, $C=C_{0}=$ const, as discussed in $\S \S 1$ and 2.3 , and are strictly valid only for thick enough films, $\bar{h}_{\infty} \gg \Gamma_{\infty} / C_{0}$ and sorption-controlled surfactant transport. In the case of thin films, $\bar{h}_{\infty} \sim \Gamma_{\infty} / C_{0}$, one must account for the variation of bulk concentration which may lead to a reversed competing effect, as suggested by the analysis of Ratulowski \& Chang (1990), and may result in a film thickening. Our study was motivated by realistic experiments with standard SDS surfactant, which parameters are outlined in $\S 1$ and justify the above assumptions. It should be noted that analogous, though much weaker, film thinning was found in a lubrication analysis of the Bretherton problem with trace amounts of surfactants by Ginley \& Radke (1989), who also assumed constant bulk concentration. However, their analysis was restricted to trace amounts and short, $\sim l_{c} C a^{1 / 3}$, dynamic meniscus versus our fully nonlinear study. In conclusion, the predicted thinning is universal under the assumptions of a thick film and sorption-controlled surfactant transport, and is insensitive to reasonable changes in other parameters, such as $P e_{s}$ and $R T \Gamma_{\infty} / \sigma_{0}$.

Our study does not confirm the effect of surfactants, that Groenveld (1970) and Park (1991) had in mind, and which is persistently invoked (e.g. Quére 1999), in order to explain the film thickening effect due to surfactants. Instead, pure hydrodynamic modelling of the surfactant effect leads to thinning, which contradicts the experimental observations for very thin films. This suggests that in the limit of very thin films (which in the case of the Landau-Levich problem is usually equivalent to low capillary numbers since $l_{c} \sim 10^{-1} \mathrm{~cm}$, but which in the case of coating a small-radius fibre can still give rise very thin films even for moderate capillary numbers), the pure hydrodynamic approach does not satisfactorily explain the observations. Therefore further research in this direction is necessary.

This work was supported by the Office of Basic Energy Sciences, US Dept of Energy.

\section{Appendix. Continuous and discrete boundary-integral formulation}

\section{A.1. Boundary-integral formulation}

We briefly outline the derivation of the two integral equations which are central in the subsequent analysis. In view of the specific region orientation, as shown in figure 3 - outward normal $\boldsymbol{n}$ and clockwise arclength $\mathrm{d} s$ - the divergence theorem for a vector-function $\boldsymbol{f}$ in a region $\Omega$ assumes the form

$$
\iint_{\Omega} \operatorname{div} \boldsymbol{f} \mathrm{d} x \mathrm{~d} y=-\int_{\partial \Omega^{-}} \boldsymbol{f} \cdot \boldsymbol{n} \mathrm{d} s,
$$

so that the second Green's identity for two functions $u(x, y)$ and $v(x, y)$, obtained by applying the divergence theorem to the vector-function $v \nabla u$ :

$$
\int_{\Omega}\{v \Delta u-u \Delta v\} \mathrm{d} q=-\oint_{\partial \Omega}\left\{v \frac{\partial u}{\partial \boldsymbol{n}}-u \frac{\partial v}{\partial \boldsymbol{n}}\right\} \mathrm{d} s(q) .
$$

Taking $v$ as a fundamental solution, i.e. Green's function $G_{l}$, of the Laplace operator, the second identity furnishes the third Green's identity,

$$
w(p) u(p)=-\int_{\Omega} G_{l} \Delta u \mathrm{~d} q-\oint_{\partial \Omega}\left\{G_{l} \frac{\partial u}{\partial \boldsymbol{n}}-u \frac{\partial G_{l}}{\partial \boldsymbol{n}}\right\} \mathrm{d} s(q), \quad w(p)=\left\{\begin{array}{cl}
1, & p \in \Omega, \\
\frac{\theta}{2 \pi}, & p \in \partial \Omega^{-} \\
0, & p \notin \bar{\Omega}
\end{array}\right.
$$


that is the solution is represented as a sum of three Newtonian (logarithmic) potentials: volume, single-layer and double-layer potentials, respectively (cf. Vladimirov 1971). Applying this identity to $u(p)=\omega(p)$, we immediately arrive at the first (for the vorticity) of the two integral equations

$$
w(p) \omega(p)=\oint_{\partial \Omega}\left\{G_{l} \frac{\partial \omega}{\partial \boldsymbol{n}}-\omega \frac{\partial G_{l}}{\partial \boldsymbol{n}}\right\} \mathrm{d} s(q),
$$

since $\omega$ is a harmonic function. In the case $u(p)=\psi(p)$, we have

$$
w(p) \psi(p)=\oint_{\partial \Omega}\left\{G_{l} \frac{\partial \psi}{\partial \boldsymbol{n}}-\psi \frac{\partial G_{l}}{\partial \boldsymbol{n}}\right\} \mathrm{d} s(q)-\int_{\Omega} \nabla \omega G_{l} \mathrm{~d} q,
$$

where the last volume integral needs to be reduced to the boundary one. Using the fact that the fundamental solution for the biharmonic equation is connected to that for the Laplace equation as $\Delta G_{b}=G_{l}$, and applying the second Green's identity (A 1) to $u=\omega$ and $v=G_{b}$, we finally obtain the second boundary-integral equation (for the streamfunction):

$$
w(p) \psi(p)=-\oint_{\partial \Omega}\left\{G_{l} \frac{\partial \psi}{\partial \boldsymbol{n}}-\psi \frac{\partial G_{l}}{\partial \boldsymbol{n}}\right\} \mathrm{d} s-\oint_{\partial \Omega}\left\{G_{b} \frac{\partial \omega}{\partial \boldsymbol{n}}-\omega \frac{\partial G_{b}}{\partial \boldsymbol{n}}\right\} \mathrm{d} s .
$$

Equations (A 2-A3) are the reduction of the original two-dimensional field problem to the one-dimensional integral equations on the boundary and serve as the basis for the boundary-element implementation.

\section{A.2. Discretization}

In the analysis of our free-interface problem we use the simplest formulation of the boundary-element method when the boundary is discretized into $N$ constant elements. Contrary to the remark of Pozrikidis (1992), the constant element formulation does allow one to define curvature and thus is applicable to such a type of problems. As known, in this formulation, the curvature is a 'virtual' variable without direct visual effect on the element shape. The points where the unknown values are considered are called 'nodes' and taken to be in the middle of the element. The values of $\psi, \psi_{n}, \omega$ and $\omega_{n}$ are assumed to be constant over each element and equal to the value at the mid-element node. Note, that for this type of element (i.e. constant) the boundary is always smooth as the node is at the centre of the element, hence the multiplier $w(p)$ is always $1 / 2$ at the boundary. Discretizing the integral equations (A 2)-(A 3), we obtain

$$
\begin{aligned}
w(p) \psi(p)= & \sum_{j=1}^{N}\left[\psi\left(q_{j}\right) \int_{\partial \Omega_{j}} \frac{\partial G_{l}}{\partial \boldsymbol{n}}\left(p, q_{j}\right) \mathrm{d} s(q)-\frac{\partial \psi}{\partial \boldsymbol{n}}\left(q_{j}\right) \int_{\partial \Omega_{j}} G_{l}\left(p, q_{j}\right) \mathrm{d} s(q)\right. \\
& \left.+\omega\left(q_{j}\right) \int_{\partial \Omega_{j}} \frac{\partial G_{b}}{\partial \boldsymbol{n}}\left(p, q_{j}\right) \mathrm{d} s(q)-\frac{\partial \omega}{\partial \boldsymbol{n}}\left(q_{j}\right) \int_{\partial \Omega_{j}} G_{b}\left(p, q_{j}\right) \mathrm{d} s(q)\right], \\
w(p) \omega(p)= & \sum_{j=1}^{N}\left[\omega\left(q_{j}\right) \int_{\partial \Omega_{j}} \frac{\partial G_{l}}{\partial \boldsymbol{n}}\left(p, q_{j}\right) \mathrm{d} s(q)-\frac{\partial \omega}{\partial \boldsymbol{n}}\left(q_{j}\right) \int_{\partial \Omega_{j}} G_{l}\left(p, q_{j}\right) \mathrm{d} s(q)\right],
\end{aligned}
$$

which allows us to recover the solution at any point $p \in \bar{\Omega}$ from the boundary data. But first we are interested in finding the solution at the nodes $q_{i}$ on the boundary. 


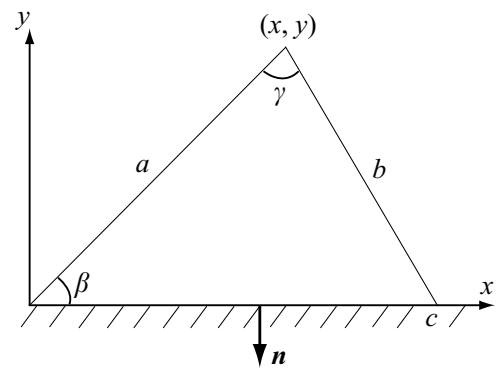

FIGURE 10. Analytical calculation of the influence coefficients.

Therefore, the above system for the boundary nodes $p=q_{i}, i=1, \ldots, N$ can be written as

$$
\begin{array}{r}
\boldsymbol{A \psi + B} \frac{\partial \psi}{\partial \boldsymbol{n}}+\boldsymbol{C} \boldsymbol{\omega}+\boldsymbol{D} \frac{\partial \boldsymbol{\omega}}{\partial \boldsymbol{n}}=0, \\
\boldsymbol{A} \boldsymbol{\omega}+\boldsymbol{B} \frac{\partial \boldsymbol{\omega}}{\partial \boldsymbol{n}}=0,
\end{array}
$$

where the matrix (influence) elements are:

$$
\begin{aligned}
A_{i j} & =f_{\partial \Omega_{j}} \frac{\partial G_{l}}{\partial \boldsymbol{n}}\left(q_{i}, q_{j}\right) \mathrm{d} s\left(q_{j}\right)-\delta_{i j} w\left(q_{j}\right), & C_{i j} & =\int_{\partial \Omega_{j}} \frac{\partial G_{b}}{\partial \boldsymbol{n}}\left(q_{i}, q_{j}\right) \mathrm{d} s\left(q_{j}\right), \\
B_{i j} & =-\int_{\partial \Omega_{j}} G_{l}\left(q_{i}, q_{j}\right) \mathrm{d} s\left(q_{j}\right), & D_{i j} & =-\int_{\partial \Omega_{j}} G_{b}\left(q_{i}, q_{j}\right) \mathrm{d} s\left(q_{j}\right) .
\end{aligned}
$$

The signs of these integrals depend on the definition of normal and boundary orientation, and are therefore provided here. The calculation of these influence coefficients can be performed analytically as in Jaswon \& Symm (1977) and Kelmanson (1983b). The integrands in the influence coefficients are the functions of the distance between points $p$ and $q$ only, so that we can perform any kind of transformation of the coordinate system involving rotations and translations. One convenient choice is shown in figure 10 , so that $\mathrm{d} s=\mathrm{d} \xi, r=\sqrt{(x-\xi)^{2}+y^{2}}, \boldsymbol{n}=-\boldsymbol{j}$ and

$$
\begin{aligned}
G_{l} & =\frac{1}{2 \pi} \ln \frac{1}{r}, & \frac{\partial G_{l}}{\partial \boldsymbol{n}} & =\frac{1}{2 \pi r^{2}} y, \\
G_{b} & =\frac{1}{8 \pi} r^{2}\left[\ln \frac{1}{r}+1\right], & \frac{\partial G_{b}}{\partial \boldsymbol{n}} & =-\frac{1}{8 \pi} y\left[2 \ln \frac{1}{r}+1\right] .
\end{aligned}
$$

The resulting expressions for the integrals in this coordinate-free representation are:

$$
\begin{aligned}
\int_{\partial \Omega_{j}} G_{l}\left(p, q_{j}\right) \mathrm{d} s & =-\frac{1}{2 \pi}\{a \cos \beta(\ln a-\ln b)+c \ln b-c+a \gamma \sin \beta\} \equiv \frac{1}{2 \pi} I, \\
\int_{\partial \Omega_{j}} G_{b}\left(p, q_{j}\right) \mathrm{d} s= & -\frac{1}{24 \pi}\left\{(c-a \cos \beta)^{3}\left(\ln b-\frac{4}{3}\right)\right. \\
& \left.+(a \cos \beta)^{3}\left(\ln a-\frac{4}{3}\right)-a^{2} \sin ^{2} \beta(3 I+2 c+\gamma a \sin \beta)\right\}, \\
f_{\partial \Omega_{j}} \frac{\partial G_{l}}{\partial \boldsymbol{n}}\left(p, q_{j}\right) \mathrm{d} s= & \frac{\gamma}{2 \pi}, \quad \int_{\partial \Omega_{j}} \frac{\partial G_{b}}{\partial \boldsymbol{n}}\left(p, q_{j}\right) \mathrm{d} s=-\frac{a \sin \beta}{8 \pi}\{c+2 I\} .
\end{aligned}
$$


The connection of the constants involved to our coordinates is established through the expressions

$$
\begin{gathered}
a=\sqrt{\left(x-\xi_{j-1 / 2}\right)^{2}+\left(y-h_{j-1 / 2}\right)^{2}}, \quad b=\sqrt{\left(x-\xi_{j+1 / 2}\right)^{2}+\left(y-h_{j+1 / 2}\right)^{2}}, \\
c=\sqrt{\left(\xi_{j+1 / 2}-\xi_{j-1 / 2}\right)^{2}+\left(h_{j+1 / 2}-h_{j-1 / 2}\right)^{2}},
\end{gathered}
$$

and the cosine theorem, $c^{2}=a^{2}+b^{2}-2 a b \cos \gamma$. The integral containing the normal derivative of $G_{l}$ has a singularity when $p=q_{i}$, i.e. for diagonal entries, but because

$$
\frac{\partial G_{l}}{\partial \boldsymbol{n}}=-\frac{1}{2 \pi r} \frac{\partial r}{\partial \boldsymbol{n}}=-\frac{1}{2 \pi r^{2}} \boldsymbol{r} \cdot \boldsymbol{n},
$$

the integral reduces to

$$
f_{\partial \Omega_{i}} \frac{\partial G_{l}}{\partial \boldsymbol{n}}\left(q_{i}, q_{i}\right) \mathrm{d} s=-\frac{1}{2 \pi} \int_{\partial \Omega_{i}} \frac{\left(\boldsymbol{q}-\boldsymbol{q}_{i}\right) \cdot \boldsymbol{n}}{\left|\boldsymbol{q}-\boldsymbol{q}_{i}\right|^{2}} \mathrm{~d} s(q),
$$

that is equal identically to zero since vectors $\boldsymbol{q}-\boldsymbol{q}_{i}$ and $\boldsymbol{n}$ are perpendicular. In this context, the remark of $\mathrm{Lu} \&$ Chang (1988) concerning the invalidity of setting this integral to zero and the necessity of special treatment is erroneous.

\section{REFERENCES}

Becker, A. A. 1992 The Boundary Element Method in Engineering. MacGraw-Hill.

Bretherton, F. P. 1961 The motion of long bubbles in tubes. J. Fluid Mech. 10, 166-189.

CARroll, B. J. \& LuCASSEN, J. 1973 Capillarity-controlled entrainment of liquid by a thin cylindrical filament moving through an interface. Chem. Engng Sci. 28, 23-30.

Deryagin, B. \& Titievskaya, A. 1945 Experimental study of liquid film thickness left on a solid wall after receeding meniscus. Dokl. Akad. Nauk USSR 50, 307-310.

Fairbrother, F. \& Stubbs, A. 1935 Studies in electroendosmosis. Part VI. The bubble-tube methods of measurements. J. Chem. Sci. 1, 527-529.

Ghadiali, S. N. \& GaVER III, D. P. 2003 The influence of non-equilibrium surfactant dynamics on the flow of a semi-infinite bubble in a rigid cylindrical capillary tube. J. Fluid Mech. 478, 165-196.

Ginley, G. M. \& RADKE, C. J. 1989 Influence of soluble surfactants on the flow of long bubbles through a cylindrical capillary. ACS Symp. Series 396, 480-501.

Goodwin, R. \& Homsy, G. M. 1991 Viscous flow down a slope in the vicinity of a contact line. Phys. Fluids 3, 515-528.

Gramlich, C. M., Mazouchi, A. \& Homsy, G. M. 2004 Time-dependent free surface Stokes flow with a moving contact line. II. Flow over wedges and trenches. Phys. Fluids 16, 1660-1667.

Groenveld, P. 1970 Low capillary number withdrawal. Chem. Engng Sci. 25, 1259-1266.

Jaswon, M. A. \& Symm, G. T. 1977 Integral Equation Methods in Potential Theory and Elastostatics. Academic.

Kelmanson, M. A. 1983a Boundary integral equation solution of viscous flows with free surfaces. J. Engng Maths 17, 329-343.

Kelmanson, M. A. $1983 \mathrm{~b}$ An integral equation method for the solution of singular slow flow problems. J. Comput. Phys. 51, 139-158.

Kistler, S. F. \& Schweizer, P. M. 1997 Liquid Film Coating: Scientific Principles and their Technological Implications. Chapman \& Hall.

Krechetnikov, R. \& Homsy, G. M. 2004 On a new surfactant-driven fingering phenomenon in a Hele-Shaw cell. J. Fluid Mech. 509, 103-124.

Krechetnikov, R. \& Homsy, G. M. 2005 Experimental study of substrate roughness and surfactant effects on the Landau-Levich law. Phys. Fluids 17, 102108.

Kuiken, H. K. 1996 Deforming surfaces and viscous sintering. In The Mathematics of Deforming Surfaces (ed. D. G. Dritschel \& R. J. Perkins), pp. 75-97. Oxford University Press. 
Ladyzhenskaya, O. A. 1963 The Mathematical Theory of Viscous Incompressible Flow. Gordon \& Breach.

Landau, L. \& Levich, B. 1942 Dragging of a liquid by a moving plate. Acta Physicochim. (USSR) $17,42-54$.

Lu, W.-Q. \& Chang, H.-C. 1988 An extension of the biharmonic boundary integral method to free surface flow in channels. J. Comput. Phys. 77, 340-360.

Mazouchi, A., Gramlich, C. M. \& Homsy, G. M. 2004 Time-dependent free surface Stokes flow with a moving contact line. I. Flow over plane surfaces. Phys. Fluids 16, 1647-1659.

Mazouchi, A. \& Homsy, G. M. 2001 Free surface Stokes flow over topography. Phys. Fluids 13, 2751-2761.

Moffatt, H. K. 1964 Viscous and resistive eddies near a sharp corner. J. Fluid Mech. 18, 1-18.

Morey, F. C. 1940 Thickness of a liquid film adhering to surface slowly withdrawn from the liquid. Natl Bur. Stan. J. Res. 25, 385-393.

PARK, C.-W. 1991 Effects of insoluble surfactants on dip coating. J. Colloid Interface Sci. 146, 382-394.

PozRIKIDIS, C. 1992 Bounday Integral and Singularity Methods for Linearized Viscous Flow. Cambridge University Press.

QuÉrÉ, D. 1999 Fluid coating on a fibre. Annu. Rev. Fluid Mech. 31, 347-384.

Quéré, D. \& DE RYCK, A. 1998 Le mouillage dynamique des fibres. Ann. Phys. Fr. 23, 1-149.

RAMdANE, O. O. \& Qú́rÉ, D. 1997 Thickening factor in Marangoni coating. Langmuir 13, 29112916.

Ratulowski, J. \& Chang, H.-C. 1990 Marangoni effects of trace impurities on the motion of long gas bubbles in capillarities. J. Fluid Mech. 210, 303-328.

RÉglat, O., Labrie, R. \& TANGUY, P. A. 1993 A new free surface model for the dip coating process. J. Comput. Phys. 109, 238-246.

Reinelt, D. A. \& Saffman, P. G. 1985 The penetration of a finger into a viscous fluid in a channel and tube. SIAM J. Sci. Stat. Comput. 6, 542-561.

RucKenstein, E. 2002 Scaling analysis of coating of a plate or a fibre. J. Colloid Interface Sci. 246, 393-400.

Saito, H. \& Scriven, L. E. 1981 Study of coating flow by the finite element method. J. Comput. Phys. 42, 53-76.

Schwartz, L. W., Princen, H. M. \& Kiss, A. D. 1986 On the motion of bubbles in capillary tubes. J. Fluid Mech. 172, 259-275.

Shen, A. Q., Gleason, B., McKinley, G. H. \& Stone, H. A. 2002 Fibre coating with surfactant solutions. Phys. Fluids 14, 4055-4068.

Shen, E. I. \& Udell, K. S. 1985 A finite element study of low Reynolds number two-phase flow in cylindrical tubes. Trans. ASME E: J. Appl. Mech. 52, 253-256.

Silliman, W. J. \& Scriven, L. E. 1980 Separating flow near a static contact line - slip at a wall and shape of a free-surface. J. Comput. Phys. 34, 287-313.

Spiers, R. P., Subbaraman, C. V. \& Wilkinson, W. L. 1974 Free coating of a Newtonian liquid onto a vertical surface. Chem. Engng Sci. 29, 389-396.

Stebe, K. J. \& BARTHÈs-Biesel, D. 1995 Marangoni effects of adsorption-desorption controlled surfactants on the leading end of an infinitely long bubble in a capillary. J. Fluid Mech. 286, $25-48$.

Stone, H. A. \& Leal, L. G. 1990 The effects of surfactants on drop deformation and breakup. J. Fluid Mech. 220, 161-186.

Tallmadge, J. A. 1967 Entrainment of liquid films. drainage, withdrawal and removal. Indust. Engng Chem. 59, 19-34.

TANGuY, P., Fortin, M. \& Choplin, L. 1984 Finite element simulation of dip coating. I. Newtonian fluids. Intl J. Numer. Meth. Fluids 4, 441-457.

TAYLOR, G. I. 1961 Deposition of a viscous fluid on the wall of a tube. J. Fluid Mech. 10, 161-165.

Vladimirov, V. S. 1971 Equations of Mathematical Physics. M. Dekker, New York.

Wassmuth, F., Laidlaw, W. G. \& Coombe, D. A. 1993 Calculation of interfacial flows and surfactant redistribution as a gas/liquid interface moves between two parallel plates. Phys. Fluids 5, 1533-1548.

Weinstein, S. J. \& Ruschak, K. J. 2004 Coating flows. Annu. Rev. Fluid Mech. 36, 29-53. 
White, D. A. \& Tallmadge, J. A. 1965 Theory of drag out of liquids on flat plates. Chem. Engng Sci. 20, 33-37.

WiLson, S. D. R. 1982 The drag-out problem in film coating theory. J. Engng Maths 16, 209221.

YoungRen, G. K. \& ACRIVOS, A. 1975 Stokes flow past a particle of arbitrary shape: a numerical method of solution. J. Fluid Mech. 69, 377-403.

Youngren, G. K. \& Acrivos, A. 1976 On the shape of gas bubble in a viscous extensional flow. J. Fluid Mech. 76, 433-442. 